\title{
Daily Life Physical Activity, Quality of Life and Symptoms of Depression and Anxiety in Adult Patients with Type 2 Diabetes: A Preliminary Study
}

\author{
Ioannis D. Morres* ${ }^{\circledR}$, Evlalia Touloudi, Antonis Hatzigeorgiadis, Athanasios Z. Jamurtas, \\ Odysseas Androutsos, Yannis Theodorakis
}

School of Physical Education, Sport Science and Dietetics, University of Thessaly, Trikala, Greece

Email: ^iomorres@pe.uth.gr

How to cite this paper: Morres, I. D., Touloudi, E., Hatzigeorgiadis, A., Jamurtas, A. Z., Androutsos, O., \& Theodorakis, Y. (2021). Daily Life Physical Activity, Quality of Life and Symptoms of Depression and Anxiety in Adult Patients with Type 2 Diabetes: A Preliminary Study. Psychology, 12, 1277-1286.

https://doi.org/10.4236/psych.2021.128080

Received: July 15, 2021

Accepted: August 20, 2021

Published: August 23, 2021

Copyright (c) 2021 by author(s) and Scientific Research Publishing Inc. This work is licensed under the Creative Commons Attribution International License (CC BY 4.0).

http://creativecommons.org/licenses/by/4.0/ (cc) (i) Open Access

\begin{abstract}
Purpose: Many diabetic patients experience depression/anxiety and poor Quality of Life (QoL). Daily life Physical Activity (PA) is linked to improved depression/anxiety and QoL across various patients, but relevant studies in diabetic patients are scarce. This preliminary study examined if daily life PA is linked to better depression/anxiety and QoL in patients with Type $2 \mathrm{Di}$ abetes (T2D). Methods: $\mathrm{A}$ total of 51 adult T2D outpatients $($ Mean age $=$ 63.31 years; Standard Deviation $=13.88$ ) completed questionnaires for PA, QoL and depression/anxiety. Descriptive and correlation statistics were computed for all variables of interest. Hierarchical regression analysis examined if days of PA at light, moderate or vigorous intensity predict improved depression/anxiety and QoL. Results: The sample was insufficiently active; also, $32 \%$ showed poor QoL, and $22 \%$ and $30 \%$, respectively, had at least moderate depression or anxiety symptoms. Days of PA at light and moderate but not at vigorous intensity demonstrated small-to-moderate inverse correlations with improved depression/anxiety and QoL. Hierarchical regression analyses demonstrated that, after controlling for age and body mass index, days of PA at light and moderate intensities predicted lower depression and explained 39\% of the variance, whereas only days of PA at light intensity predicted lower anxiety and better QoL, explaining, respectively, $30 \%$ and $40 \%$ of the variance. Discussion: This preliminary study for adults with T2D found that days of light and moderate intensity PA were linked to improved depression, but days of only light intensity PA were linked to improved anxiety and QoL. Findings are encouraging, especially since our sam-
\end{abstract}


ple was insufficiently active. However, larger samples with T2D adults are needed for firmer conclusions.

\section{Keywords}

Daily Life Physical Activity, Diabetes, Depression, Anxiety, Quality of Life, Adults

\section{Introduction}

Diabetes is a chronic medical condition, in which body cells cannot activate hormone insulin to move increased glucose from blood. In the US, $10.5 \%$ of adults are diabetic (Centers for Disease Control and Prevention, 2020), with 90\% to $95 \%$ being diagnosed with Type 2 Diabetes (T2D). All T2D treatments require intensive blood sugar monitoring. In light of this challenge, blood glucose is often dysregulated, causing physical health diseases (e.g., cardiovascular or kidney diseases). Hence, diabetic patients report depression and anxiety twice as much as the general population (Anderson, Freedland, Clouse, \& Lustman, 2001), and poor quality of life (QoL) (Daniele, Bruin, Oliveira, Pompeu, \& Forti, 2013; Wändell, Brorsson, \& Åberg, 1997). Easily-operated effective interventions are thus needed to improve depression/anxiety and QoL in T2D patients.

Physical exercise shows antidepressant and anxiolytic effects in clinical settings across various patients (Gordon, McDowell, Lyons, \& Herring, 2017; Morres, Hatzigeorgiadis, Stathi, et al., 2019) including diabetic patients (e.g., Narita, Inagawa, Stickley, \& Sugawara, 2019). However, exercise shows high dropouts in pragmatic settings (Crone, Johnston, Gidlow, Henley, \& James, 2008). This is possibly due to increased operational demands, for example, the operation of exercise on prescription for depression demands group- and supervised-based moderate intensity exercise of $45 \mathrm{~min} / \mathrm{session}, 3$ sessions/week for 10 - 14 weeks, in sport and leisure facilities such as gyms (National Institute for Clinical Excellence [NICE], 2009). Alternatives to exercise with less operational demands are thus essential. Daily life physical activity (PA) is considered an alternative because it is associated with better mental health through the single operational demand of $\geq 150 \mathrm{~min} /$ week at moderate intensity (World Health Organization [WHO], 2020). Daily life PA has been related with improved mental health indices including depression or anxiety across various groups (Meira, Meneguelli, Leopoldo, \& Florindo, 2020; Morres, Hatzigeorgiadis, Krommidas, et al., 2019), but relevant studies in exclusively diabetic patients are limited (Kim, 2018) and highly essential (Cezaretto, Ferreira, Sharma, Sadeghirad, \& Kolahdooz, 2016). In light of the lack of previous research exploring the mental health correlators of daily life PA in T2D adult patients, this study examined whether daily life PA predicts improved depressive/anxiety symptoms and QoL in mature-adult patients with T2D. 


\section{Methodology}

\subsection{Participants}

A total of 51 adults ( 우 56.9\%) with T2D were recruited from a primary health unit for diabetes treatment within a period of 12 months. The mean age was 63.31 years; Standard Deviation $(\mathrm{SD})=13.88$ (age range $34-93$ years). The mean Body Mass Index (BMI) was 28.14; $\mathrm{SD}=4.04$, indicating that the sample was overweight.

\subsection{Measures}

The Godin-Shephard Leisure-Time PA Questionnaire (GSLTPAQ) (Godin \& Shephard, 1985) was employed to assess Leisure Time PA (LTPA). This is a self-administrated 3-item scale. The three items record the weekly frequency a person participates in mild, moderate and vigorous LTPA for $\geq 15$ minutes (each item includes examples of LTPA intensities). The total score of LTPA (defined as Leisure Score Index [LSI]), is the sum of the light, moderate and vigorous intensity PA frequencies multiplied, respectively, by the metabolic equivalents of 3, 5, and 9. With respect to health-related PA levels, the GSLTPAQ suggests that moderate-to-vigorous LSI of $\geq 24$ or $\leq 23$ correspond to active or insufficiently active lifestyle, respectively (Godin, 2011). The GSLTPAQ has been used in the Greek population (Bebetsos, Chasandra, \& Matsouka, 2020).

Depression

The Patient Health Questionnaire-9 (PHQ-9) was employed to assess depression (Kroenke, Spitzer, \& Williams, 2001). This self-report 9-item questionnaire assesses depressive symptom severity over the past two weeks on a 4-point severity scale. Total scoring ranges from 0 to 27 , with higher scores indicating more severe symptoms of depression (relevant cutoff scores: $0-4=$ minimal or none; 5 - 9 = mild; 10 - $14=$ moderate; 15 - $19=$ moderately severe; $20-27=$ severe). A clinical condition and psychometric diagnosis of depression are considered when a score of $\geq 2$ is recorded in items 1 or 2 and in five of the remaining seven items excluding item 9 which may also record a score of $\geq 1$. The PHQ-9 has been previously used in the Greek population (Hyphantis et al., 2011).

Depression and Anxiety

Depression and anxiety symptoms were assessed with the self-report Hospital Anxiety and Depression scale (HADS) (Zigmond \& Snaith, 1983). This 14-item questionnaire includes two 7-point subscales measuring anxiety or depressive symptom severity. Answers are organized on a 4-point Likert scale ranging from 0 to 3, with higher scores indicating more severe symptoms. The total score of HADS is the sum of the 14 items, and for each subscale the score is the sum of the corresponding seven items (ranging from $0-21$ ). This tool has been used in the Greek population (Michopoulos et al., 2008).

Quality of Life

The SF-12 Health Survey was employed to assess health related QoL (Ware, Kolinski, \& Keller, 1996). There is a self-report questionnaire with items scoring 
on a Likert scale. This questionnaire is a widely used to assess health related QoL aspects referred to physical and mental health. These aspects include physical limitations, dysfunction due to physical health problems or due to emotional health problems, social functioning, emotional wellbeing, energy, pain, fatigue, and global health perceptions. Higher scoring indicates better quality of life. A score of $<35$ indicates poor QoL. The SF-12 has been previously used in the Greek population (Kontodimopoulos, Pappa, Niakas, \& Tountas, 2007).

\subsection{Statistical Analysis}

Means and SDs, Cronbach's Alpha and spearman correlation coefficients were computed for all variables of interest. Three hierarchical regressions analyses examined the degree to which days of PA at light, moderate or vigorous intensity could predict QoL, anxiety, and depression. In all analyses, age and BMI were entered in the first step to account for the effect of age and BMI on the dependent variables, which were identified through the correlation analysis, whereas days of PA at low, moderate or vigorous intensity were entered in the second step.

\section{Results}

Descriptive statistics, Cronbach's alpha coefficients for the scales are presented in Table 1. Correlations between all variables are presented in Table 2. The

Table 1. Descriptive statistics, reliability coefficients and correlations $(\mathrm{N}=51)$.

\begin{tabular}{cccc}
\hline & \multicolumn{2}{c}{ Descriptives } & \multirow{2}{*}{ Cronbach's Alpha } \\
\cline { 2 - 4 } & Mean & Standard Deviation & \\
\hline Quality of Life & 38.47 & 9.71 & 0.93 \\
Anxiety & 8.27 & 5.28 & 0.89 \\
Depression & 6.90 & 4.43 & \\
Light intensity PA (days) & 3.45 & 2.02 & \\
Moderate intensity PA (days) & 1.80 & 2.20 & \\
Vigorous intensity PA (days) & 0.49 & 1.35 & \\
\hline
\end{tabular}

PA: Physical Activity.

Table 2. Correlation coefficients.

\begin{tabular}{|c|c|c|c|c|c|}
\hline & 1 & 2 & 3 & 4 & 5 \\
\hline \multicolumn{6}{|l|}{ 1. Quality of Life } \\
\hline 2. Anxiety & $-0.70^{\star *}$ & & & & \\
\hline 3. Depression & $-0.82^{\star *}$ & $0.71^{\star *}$ & & & \\
\hline 4. Light intensity PA (days) & $0.61^{* *}$ & $-0.47^{\star *}$ & $-0.55^{\star *}$ & & \\
\hline 5. Moderate intensity PA (days) & $0.48^{\star *}$ & $-0.29^{*}$ & $-0.49^{\star *}$ & $0.55^{\star *}$ & \\
\hline 6. Vigorous intensity PA (days) & 0.16 & 0.04 & -0.04 & 0.15 & $0.50^{* *}$ \\
\hline
\end{tabular}


reliability of all scales was satisfactory, ranging from 0.85 to 0.93 . In one out of three participants (32\%) the mean score for QoL was $<35$ indicating poor QoL. The PHQ-9 algorithms indicated that $25 \%$ of the participants recorded a score that corresponded to a psychometric diagnosis of major depression. The HADS algorithms indicated that the average scores for anxiety and depression were low to moderate; also, $22 \%$ and $30 \%$ of the participants recorded at least moderate scores in depression and anxiety $(\geq 11)$, respectively. Based on the GSLTPAQ (Godin, 2011), participants reported on average 3.45 days of light, 1.8 days of moderate and 0.5 days of vigorous intensity PA per week. The GSLTPAQ average unit calculated from all PA intensities rated our sample as moderately active to active (Mean of GSLTPAQ unit = 23.78; SD = 23.17). The health-related average unit of GSLTPAQ (calculated from moderate and vigorous PA intensities), rated our sample as insufficiently active (Mean of health-related GSLTPAQ unit $=13.43$; SD $=20.09$ ). Finally, days of PA at moderate and light but not at vigorous intensity showed moderate negative correlations with anxiety and depression and moderate positive correlations with QoL.

The first hierarchical regression treated QoL as a dependent variable. In the first step of the analysis, age and BMI predicted $11.3 \%$ of the QoL variance, F (2, $48)=3.04, p=0.05$. Only BMI (beta $=-0.28$ ) contributed significantly to the prediction of QoL. In the second step, the introduction of PA raised the prediction to $40 \%$ of the variance, $\mathrm{F}(2,45)=6.04, p<0.001$. In this step, days of PA at light intensity were the stronger predictor of QoL (beta $=0.46)$. Results are presented in Table 3 . The second hierarchical regression treated anxiety scores (from HADS subscale) as a dependent variable. In the first step of the analysis, age and BMI predicted $5.5 \%$ of the anxiety variance, however, the prediction was not significant, $\mathrm{F}(2,48)=1.39, p=0.26$. In the second step, the introduction of $\mathrm{PA}$ raised the prediction to $30.1 \%$ of the variance, $\mathrm{F}(2,45)=3.88, p<0.01$. In this step, days of PA at light intensity were the stronger (beta $=-0.33$ ) and sole significant predictor of anxiety. Results are presented in Table 4. The third hierarchical regression treated depression (from HADS subscale) as a dependent variable. In the first step of the analysis, age and BMI predicted $12.9 \%$ of the

Table 3. Regression analysis for quality of life (SF-12).

\begin{tabular}{|c|c|c|c|c|c|c|}
\hline & & $\mathrm{R}^{2}$ & $\mathrm{~F}$ & beta & $\mathrm{t}$ & $p$ \\
\hline \multirow[t]{3}{*}{ Step 1} & & 0.112 & 3.04 & & & \\
\hline & Age & & & -0.193 & -1.42 & 0.163 \\
\hline & Body mass index & & & -0.281 & -2.07 & 0.044 \\
\hline \multirow[t]{4}{*}{ Step 2} & & 0.401 & 6.04 & & & \\
\hline & Light intensity PA (days) & & & 0.468 & 3.177 & 0.003 \\
\hline & Moderate intensity PA (days) & & & 0.214 & 1.300 & 0.200 \\
\hline & Vigorous intensity PA (days) & & & -0.028 & -0.204 & 0.840 \\
\hline
\end{tabular}

PA: Physical Activity. 
Table 4. Regression analysis for anxiety (HADS-anxiety).

\begin{tabular}{|c|c|c|c|c|c|c|}
\hline & & $\mathrm{R}^{2}$ & $\mathrm{~F}$ & beta & $\mathrm{t}$ & $p$ \\
\hline \multirow[t]{3}{*}{ Step 1} & & 0.055 & 1.390 & & & \\
\hline & Age & & & -0.099 & -0.704 & 0.485 \\
\hline & Body mass index & & & 0.208 & 1.484 & 0.144 \\
\hline \multirow[t]{4}{*}{ Step 2} & & 0.301 & 3.882 & & & \\
\hline & Light intensity PA (days) & & & -0.473 & -2.968 & 0.005 \\
\hline & Moderate intensity PA (days) & & & -0.150 & -0.844 & 0.403 \\
\hline & Vigorous intensity PA (days) & & & 0.183 & 1.248 & 0.219 \\
\hline
\end{tabular}

PA: Physical Activity.

depression variance, $\mathrm{F}(2,48)=3.54, p<0.05$. In the second step, the introduction of $\mathrm{PA}$ raised the prediction to $39 \%$ of the variance, $\mathrm{F}(2,45)=5.77, p<0.01$. In this step, days of PA at moderate (beta $=-0.37$ ) and light (beta $=-0.33$ ) intensity were the stronger and significant predictors of depression. Results are presented in Table 5.

\section{Discussion}

This study has found that daily life PA at light intensity for 3.45 days/week and at moderate intensity for 1.8 days/week predicted improved depression in T2D mature adults. Only light intensity PA predicted improved anxiety and QoL. The antidepressant predictive effects of light and moderate intensity PA in our study is partially replicating previous studies such as the study from Morres, Hatzigeorgiadis, Krommidas, et al. (2019), in which moderate, but not light, intensity PA predicted lower depression in major depressed middle-aged adult patients. Light intensity PA may thus represent an equally vital antidepressant factor to moderate intensity PA in T2D mature adults. The antidepressant impact of both PA intensities receives additional merit, as our sample was insufficiently active and $25 \%$ showed depression algorithms corresponding to a psychometrically diagnosed major depression. Hence, the promotion of PA could consider that even insufficient daily life PA levels at low or moderate intensity may contribute to lower depression in T2D patients. Light rather than moderate intensity warrants further attention due to links to decreased risk for injuries.

Light but not moderate intensity daily life PA predicted lower anxiety in our sample. This finding is not in concordance to the mainstream notion given that moderate intensity PA is widely recommended for better mental health (WHO, 2020). However, the anxiolytic effect of low intensity PA in various populations including T2D mature adults has been repeatedly recorded. For example, the Bartholomew and Linder (1998) trial demonstrated that light intensity PA decreased state anxiety, whereas moderate intensity PA increased state anxiety. Also, meta-analytic studies have reported important anxiolytic effects for light rather than for moderate intensity PA (Wipfli, Rethorst, \& Landers, 2008). It is, 
Table 5. Regression analysis for depression (HADS-depression).

\begin{tabular}{ccccccc}
\hline & & $\mathrm{R}^{2}$ & $\mathrm{~F}$ & beta & $\mathrm{t}$ & $p$ \\
\hline Step 1 & Age & 0.129 & 3.540 & & & \\
& Body mass index & & & 0.198 & 1.468 & 0.146 \\
& & & & 0.306 & 2.272 & 0.028 \\
\hline Step 2 & Light intensity PA (days) & & & -0.328 & -2.208 & 0.032 \\
& Moderate intensity PA (days) & & & -0.374 & -2.255 & 0.029 \\
& Vigorous intensity PA (days) & & & 0.205 & 1.494 & 0.142 \\
\hline
\end{tabular}

PA: Physical Activity.

therefore, important to consider a non-anxiety inducing social milieu and environmental setting when choosing T2D adults to participate in daily life PA. Apart from anxiolytic predictive effects, daily life PA at light intensity showed also predictive effects on better QoL. This finding is highlighted by the fact that T2D patients experience lower levels of QoL compared to the general population (Daniele et al., 2013; Wändell et al., 1997). In line, 32\% of our sample showed an average score in the SF-12 that was indicative of a QoL that was poor and below the general population norms (Ware et al., 1996).

The prediction of improved anxiety/depression and QoL by daily life PA at mainly light intensity should be interpreted in combination to a 2 -fold consideration. First, daily life PA is linked to mental health through the single operational demand of the accumulation of $\geq 150 \mathrm{~min} /$ week of moderate intensity (WHO, 2020). In contrast, exercise, which is widely linked to and recommended for better depression/anxiety and QoL, shows high dropouts possibly due to increased operational demands. Second, we found that daily life PA predicted improved depression/anxiety and QoL although our sample was insufficiently active lifestyle according to the GSLTPA algorithms. Both considerations suggest the multiple benefits of daily life PA, given that daily life PA in our study was related with improved disease specific symptoms such as depression and anxiety and, at the same time, with improved wellbeing indices such as quality of life. Both considerations provide us also with the opportunity to speculate that even low levels of daily life PA at mainly low intensity could be potentially emerged as a more sustainable strategy for improved depression/anxiety and QoL in comparison to exercise. This speculation highlights, in turn, the need to take into account various practical aspects. These aspects are mainly referring to the need to create and satisfy applicable practicalities for giving the opportunity to perform daily life PA at light intensity. Applicable practicalities related to daily life PA include fast track accessibility to recourses such as leisure time facilities adapted to the needs/preferences of T2D adults.

\section{Conclusion}

The findings of this study revealed a beneficial association of daily life PA with 
better QoL and improved depression and anxiety. Daily life PA in particular at low intensity, seemed to be playing a key role in predicting lower symptoms of depression and anxiety and better QoL. Based on the study findings, the scientific community may consider that various social implications are emerged including provision of T2D adults with applicable opportunities for light intensity PA participation in daily life. Our study findings, however, need to be carefully interpreted because the sample size was small. Also, the cross-sectional design of the study does not enable cause and effect interpretations. Future research should therefore examine the antidepressant/anxiolytic and QoL effects of daily life PA through randomized controlled trials in larger samples of T2D adults. In this vein, firmer conclusions will be drawn.

\section{Conflicts of Interest}

The authors declare no conflicts of interest regarding the publication of this paper.

\section{References}

Anderson, R. J., Freedland, K. E., Clouse, R. E., \& Lustman, P. J. (2001). The Prevalence of Comorbid Depression in Adults with Diabetes. Diabetes Care, 24, 1069-1078. https://doi.org/10.2337/diacare.24.6.1069

Bartholomew, J. B., \& Linder, D. E. (1998). State Anxiety Following Resistance Exercise: The Role of Gender and Exercise Intensity. Journal of Behavioral Medicine, 21, 205-219. https://doi.org/10.1023/A:1018732025340

Bebetsos, E., Chasandra, A., \& Matsouka, O. (2020). The Relationship of Attitudes and Behaviors with Working Conditions and Physical Activity among Workers in Public Hospitals in Greece. Archives of Hellenic Medicine/Arheia Ellenikes Iatrikes, 37, 457-463.

Centers for Disease Control and Prevention (2020). National Diabetes Statistics Report. Centers for Disease Control and Prevention, US Department of Health and Human Services.

Cezaretto, A., Ferreira, S. R. G., Sharma, S., Sadeghirad, B., \& Kolahdooz, F. (2016). Impact of Lifestyle Interventions on Depressive Symptoms in Individuals At-Risk of, or with, Type 2 Diabetes Mellitus: A Systematic Review and Meta-Analysis of Randomized Controlled Trials. Nutrition, Metabolism and Cardiovascular Diseases, 26, 649-662. https://doi.org/10.1016/j.numecd.2016.04.009

Crone, D., Johnston, L. H., Gidlow, C., Henley, C., \& James, D. V. B. (2008). Uptake and Participation in Physical Activity Referral Schemes in the UK: An Investigation of Patients Referred with Mental Health Problems. Issues in Mental Health Nursing, 29, 1088-1097. https://doi.org/10.1080/01612840802319837

Daniele, T. M. d. C., Bruin, V. M. S. d., Oliveira, D. S. N. d., Pompeu, C. M. R., \& Forti, A. C. (2013). Associations among Physical Activity, Comorbidities, Depressive Symptoms and Health-Related Quality of Life in Type 2 Diabetes. Arquivos Brasileiros de Endocrinologia \& Metabologia, 57, 44-50. https://doi.org/10.1590/S0004-27302013000100006

Godin, G. (2011). The Godin-Shephard Leisure-Time Physical Activity Questionnaire. The Health \& Fitness Journal of Canada, 4, 18-22. https://doi.org/10.14288/hfjc.v4i1.82 
Godin, G., \& Shephard, R. (1985). A Simple Method to Assess Exercise Behavior in the Community. Canadian Journal of Applied Sport Sciences, 10, 141-146.

Gordon, B. R., McDowell, C. P., Lyons, M., \& Herring, M. P. (2017). The Effects of Resistance Exercise Training on Anxiety: A Meta-Analysis and Meta-Regression Analysis of Randomized Controlled Trials. Sports Medicine, 47, 2521-2532.

https://doi.org/10.1007/s40279-017-0769-0

Hyphantis, T., Kotsis, K., Voulgari, P. V., Tsifetaki, N., Creed, F., \& Drosos, A. A. (2011). Diagnostic Accuracy, Internal Consistency, and Convergent Validity of the Greek Version of the Patient Health Questionnaire 9 in Diagnosing Depression in Rheumatologic Disorders. Arthritis Care \& Research, 63, 1313-1321. https://doi.org/10.1002/acr.20505

Kim, D.-J. (2018). Effects of Physical Activity on Depression in Adults with Diabetes. Osong Public Health and Research Perspectives, 9, 143-149. https://doi.org/10.24171/j.phrp.2018.9.4.02

Kontodimopoulos, N., Pappa, E., Niakas, D., \& Tountas, Y. (2007). Validity of SF-12 Scores in a Greek General Population. Health and Quality of Life Outcomes, 5, Article No. 55. https://doi.org/10.1186/1477-7525-5-55

Kroenke, K., Spitzer, R. L., \& Williams, J. B. (2001). The PHQ-9: Validity of a Brief Depression Severity Measure. Journal of General Internal Medicine, 16, 606-613. https://doi.org/10.1046/j.1525-1497.2001.016009606.x

Meira, C. M., Meneguelli, K. S., Leopoldo, M. P. G., \& Florindo, A. A. (2020). Anxiety and Leisure-Domain Physical Activity Frequency, Duration, and Intensity during Covid-19 Pandemic. Frontiers in Psychology, 11, Article No. 603770.

https://doi.org/10.3389/fpsyg.2020.603770

Michopoulos, I., Douzenis, A., Kalkavoura, C., Christodoulou, C., Michalopoulou, P., Kalemi, G., Finet, K., Patapis, P., Protopapa, K., \& Lykouras, L. (2008). Hospital Anxiety and Depression Scale (HADS): Validation in a Greek General Hospital Sample. Annals of General Psychiatry, 7, Article No. 4. https://doi.org/10.1186/1744-859X-7-4

Morres, I. D., Hatzigeorgiadis, A., Krommidas, C., Comoutos, N., Sideri, E., Ploumpidis, D., Economou, M., \& Theodorakis, Y. (2019). Objectively Measured Physical Activity and Depressive Symptoms in Adult Outpatients Diagnosed with Major Depression. Clinical Perspectives. Psychiatry Research, 280, Article ID: 112489.

https://doi.org/10.1016/j.psychres.2019.112489

Morres, I. D., Hatzigeorgiadis, A., Stathi, A., Comoutos, N., Arpin-Cribbie, C., Krommidas, C., \& Theodorakis, Y. (2019). Aerobic Exercise for Adult Patients with Major Depressive Disorder in Mental Health Services: A Systematic Review and Meta-Analysis. Depression and Anxiety, 36, 39-53. https://doi.org/10.1002/da.22842

Narita, Z., Inagawa, T., Stickley, A., \& Sugawara, N. (2019). Physical Activity for Diabetes-Related Depression: A Systematic Review and Meta-Analysis. Journal of Psychiatric Research, 113, 100-107. https://doi.org/10.1016/j.jpsychires.2019.03.014

National Institute for Clinical Excellence [NICE]. (2009). Depression in Adults: Recognition and Management. NICE Guidelines No. CG90, National Institute for Clinical Excellence.

Wändell, P. E., Brorsson, B., \& Åberg, H. (1997). Quality of Life in Diabetic Patients Registered with Primary Health Care Services in Sweden. Scandinavian Journal of Primary Health Care, 15, 97-102. https://doi.org/10.3109/02813439709018495

Ware, J. E., Kolinski, M., \& Keller, S. D. (1996). A 12-Item Short-Form Health Survey: Construction of Scales and Preliminary Tests of Reliability and Validity. Medical Care, 34, 220-233. https://doi.org/10.1097/00005650-199603000-00003

Wipfli, B., Rethorst, C., \& Landers, D. (2008). The Anxiolytic Effects of Exercise: A Me- 
ta-Analysis of Randomized Trials and Dose-Response Analysis. Journal of Sport \& EXercise Psychology, 30, 392-410. https://doi.org/10.1123/jsep.30.4.392

World Health Organization (2020). WHO Guidelines on Physical Activity and Sedentary Behaviour: At a Glance. World Health Organization.

Zigmond, A. S., \& Snaith, R. P. (1983). The Hospital Anxiety and Depression Scale. Acta Psychiatrica Scandinavica, 67, 361-370.

https://doi.org/10.1111/j.1600-0447.1983.tb09716.x 